\title{
Longitudinal Imaging of Cancer Cell Metastases in Two Preclinical Models: A Correlation of Noninvasive Imaging to Histopathology
}

\author{
Pavan P. Adiseshaiah, ${ }^{1}$ Nimit L. Patel, ${ }^{2}$ Lilia V. Ileva, ${ }^{2}$ Joseph D. Kalen, ${ }^{2}$ \\ Diana C. Haines, ${ }^{3}$ and Scott E. McNeil ${ }^{1}$ \\ ${ }^{1}$ Nanotechnology Characterization Laboratory, Cancer Research Technology Program, Leidos Biomedical Research, \\ Inc., Frederick National Laboratory for Cancer Research, Frederick, MD 21702, USA \\ ${ }^{2}$ Small Animal Imaging Program, Laboratory Animal Science Program, Leidos Biomedical Research, \\ Inc., Frederick National Laboratory for Cancer Research, Frederick, MD 21702, USA \\ ${ }^{3}$ Pathology/Histotechnology Laboratory, Laboratory Animal Science Program, Leidos Biomedical Research, \\ Inc., Frederick National Laboratory for Cancer Research, Frederick, MD 21702, USA \\ Correspondence should be addressed to Pavan P. Adiseshaiah; adiseshaiahp@mail.nih.gov
}

Received 3 December 2013; Accepted 18 January 2014; Published 3 March 2014

Academic Editor: Domenico Rubello

Copyright (C) 2014 Pavan P. Adiseshaiah et al. This is an open access article distributed under the Creative Commons Attribution License, which permits unrestricted use, distribution, and reproduction in any medium, provided the original work is properly cited.

\begin{abstract}
Metastatic spread is the leading cause of death from cancer. Early detection of cancer at primary and metastatic sites by noninvasive imaging modalities would be beneficial for both therapeutic intervention and disease management. Noninvasive imaging modalities such as bioluminescence (optical), positron emission tomography (PET)/X-ray computed tomography (CT), and magnetic resonance imaging (MRI) can provide complementary information and accurately measure tumor growth as confirmed by histopathology. Methods. We validated two metastatic tumor models, MDA-MD-231-Luc and B16-F10-Luc intravenously injected, and 4T1-Luc cells orthotopically implanted into the mammary fat pad. Longitudinal whole body bioluminescence imaging (BLI) evaluated metastasis, and tumor burden of the melanoma cell line (B16-F10-Luc) was correlated with (PET)/CT and MRI. In addition, ex vivo imaging evaluated metastasis in relevant organs and histopathological analysis was used to confirm imaging. Results. BLI revealed successful colonization of cancer cells in both metastatic tumor models over a 4-week period. Furthermore, lung metastasis of B16-F10-Luc cells imaged by PET/CT at week four showed a strong correlation $\left(R^{2}=0.9\right)$ with histopathology. The presence and degree of metastasis as determined by imaging correlated $\left(R^{2}=0.7\right)$ well with histopathology findings. Conclusions. We validated two metastatic tumor models by longitudinal noninvasive imaging with good histopathology correlation.
\end{abstract}

\section{Introduction}

Metastasis of cancer cells from primary tumors is one of the leading causes of poor prognosis and death from the disease. Metastatic spread is the root of approximately $90 \%$ of cancerrelated deaths [1]. Early detection of cancer cell metastasis would be incredibly beneficial for timely therapeutic intervention and management of the disease.

In most preclinical animal studies, gross examination and histopathological analysis are commonly used to evaluate dissemination of cancer cells to secondary sites. These techniques are limited, however, as they cannot be used to adequately monitor tumor development. Furthermore, optical microscopy may have an inadequate field of view (3D morphometric) for thorough tumor evaluation. Noninvasive imaging provides the ability to perform real-time serial imaging to enhance the observation of disease progression and/or evaluate therapeutic treatment response. In addition, noninvasive imaging allows researchers to use fewer animals with greater statistical power.

Orthotopic and metastatic tumor models provide an appropriate tumor microenvironment (seed and soil hypothesis) to mimic tumor growth observed in the clinic [2]. In addition, these models can be appropriate to study complex 
phenomena such as angiogenesis, metastasis, and invasion of cancer cells [3]. The ability to understand these complex events by noninvasive imaging modalities can help dictate proper therapeutic treatments. A number of orthotopic and metastatic tumor models utilizing the luciferase gene have been developed [4-6]. Bioluminescence imaging (BLI) using orthotopic and metastatic models enables investigators to successfully visualize tumor growth in longitudinal studies and evaluate therapeutic responses at metastatic sites. In addition, the substrate for bioluminescence imaging, Dluciferin, requires oxygen and ATP, thereby directly correlating the presence of viable cells in a tumor to luminescence and decreased luminescence to regions of hypoxia/necrosis and/or effect of chemotherapy within a tumor.

Several novel molecular imaging probes are currently being developed for noninvasive imaging technologies such as positron emission tomography (PET), computed tomography (CT), and magnetic resonance imaging (MRI) to monitor cancer progression and inflammation [7-12]. These imaging modalities have enhanced the clinicians' ability to visualize lesions with improved specificity and sensitivity, intervene with appropriate therapies, and monitor therapeutics' effects noninvasively. In addition to these clinical imaging modalities, bioluminescence and fluorescence imaging are useful methods to monitor tumor growth in primary and metastatic sites in preclinical cancer models. The high sensitivity and stability of luciferase expression in transgene cells (stable over 40 passages) correlate very well with histopathology in xenograft and orthotopic implantation models [13-15]. For example, BLI of liver metastasis of MC38 luciferaseexpressing colon cancer cells correlated well with ultrasound imaging [15].

Noninvasive imaging provides an ideal tool to monitor efficacy of chemotherapeutic agents in different tumor models [16-18]. For example, BLI and $\left[{ }^{18} \mathrm{~F}\right]$ FDG PET/CT were used to monitor tumor size of human hepatocellular carcinoma cells (HCC-LM3-fluc cells) subcutaneously implanted in Balb/c nude mice, treated with cyclophosphamide or saline vehicle control [16]. In another study, noninvasive BLI of orthotopically implanted gastric cancer cells (SGC7901-Luc) demonstrated decreased signal intensity following docetaxel treatment in comparison to a saline control, correlating therapeutic response analyzed by BLI to tumor weight [19]. In addition, BLI signal strongly correlated with tumor volume measurement in an orthotopic bladder cancer model as determined by MRI using the gadolinium-based contrast agent, Magnevist [13].

In the present study, we validated two metastatic models utilizing noninvasive imaging techniques and correlated the tumor burden using histopathology. The first model (experimental metastasis) utilized two luciferase-expressing cell lines, the human breast cancer MDA-MB-231-Luc and the murine melanoma cancer B16-F10-Luc cells, injected intravenously (i.v.) into the tail-vein. The human breast and murine melanoma cancer cells used in the present study are relevant in evaluating lung metastases, as clinically cells from both types of cancers are known to metastasize to the lung in addition to other organs. Furthermore, the experimental metastasis model provides a quick approach to evaluating tumor growth at metastatic sites and is well defined for several cancer cell lines $[5,20,21]$. In addition to BLI, we employed the radiopharmaceutical $\left[{ }^{18} \mathrm{~F}\right]$ FDG PET (glucose analog) to evaluate metabolic activity of the metastatic lesions and T2W-MRI for anatomical tumor location, which are difficult to identify by BLI alone. The second model involved an orthotopic implantation of murine mammary adenocarcinoma cancer cells, 4T1-Luc cells, with longitudinal imaging of tumor progression using whole-body bioluminescence in the presence or absence of an intact primary tumor. The $4 \mathrm{~T} 1$ cells, a triple negative mammary cancer cell line, were used as cell proliferation and the metastatic potential of this cell line mimics human breast cancer growth in a clinical setting. In addition, we implanted these cells in a syngeneic mouse model to evaluate the role of immune cells during cancer growth, spread, and therapeutic intervention. Results obtained from noninvasive imaging of the 4T1-Luc tumor model correlated to metastatic lesions identified by ex vivo imaging of resected organs and by histopathology.

\section{Methods and Materials}

2.1. Cancer Cell Lines. Luciferase-expressing cancer cell lines (human breast cancer cells MDA-MB-231-Luc, murine melanoma cancer cells B16-F10- Luc, and murine mammary cancer cells 4T1-Luc) were obtained from Caliper Life Sciences (Hopkinton, MA). Detailed construction of the lentiviral vector system, transfection, and characterization of stable cell lines were previously reported [22, 23]. All three cell lines were cultured in RPMI 1640 medium (HyClone, UT) supplemented with $10 \%$ heat inactivated fetal bovine serum (HyClone, UT) without antibiotics and tested negative for human and rodent pathogens prior to initiation of the animal studies. A single cancer cell suspension was prepared for tumor induction in Hanks Balanced Salt Solution with greater than $95 \%$ viability as evaluated by trypan blue staining.

2.2. Animal Models and Cancer Cell Implantation. Animal care was in accordance with the procedures outlined in the Guide for Care and Use of Laboratory Animals (National Research Council, 1996; National Academy Press, Washington, DC) and animal protocols were approved by the Frederick National Laboratory for Cancer Research Institutional Animal Care and Use Committee. Mouse strains used in the study were female athymic nu/nu (MDA-MB-231-Luc cells), female C57 BL/6 albino (B16-F10-Luc cells), and female Balb/c albino (4T1-Luc cells) (Charles River Laboratory, Frederick, MD), 7-9 weeks of age at procurement, and allowed to acclimate for one week prior to cell implantation.

2.2.1. Intravenous Experimental Metastatic Model. Female athymic nude $(n=5)$ and C57 BL/6 albino $(n=5)$ mice were physically restrained and intravenously injected into the tail-vein with $2 \times 10^{6} \mathrm{MDA}-\mathrm{MB}-231$-Luc cells and $1 \times 10^{5} \mathrm{~B} 16$ F10-Luc cells, respectively, in $0.1 \mathrm{~mL}$ of Hanks Balanced Salt Solution (HBSS) using a 25-gauge needle. 
2.2.2. Mammary Fat Pad Spontaneous Metastasis Model. $\mathrm{Balb} / \mathrm{c}$ mice $(n=10)$ were anesthetized with $3 \%$ isoflurane with oxygen carrier gas at $1 \mathrm{~L} / \mathrm{min}$ flow rate delivered by nosecone before the injection. The site of cancer cell injection was prepped with alcohol followed by Betadine, and a $5 \mathrm{~mm}$ incision was made anterior to the rear leg to expose the number 4 inguinal mammary gland fat pad. 4T1-Luc cells were implanted in anesthetized animals $5 \times 10^{4}$ with a total volume of $50 \mu \mathrm{L}$ HBSS through a 27 -gauge needle. Surgically exposing to the fat pad ensured correct injection of cancer cells by visual confirmation of fat pad swelling. A solution of $0.25 \%$ bupivacaine was applied to the incision before closing with a sterile wound clip. In a subgroup of animals $(n=5)$, the growth of the primary tumor was measured using calipers and surgically resected when the tumor reached $10 \mathrm{~mm}$ in diameter.

2.2.3. Bioluminescence Imaging (BLI). In vitro BLI was performed according to manufacturer's procedures (IVIS SPECTRUM, PerkinElmer Inc., Waltham, MA). Correlation of the BLI signal intensity with the number of cells and optimal imaging time (based on peak luciferin uptake) was determined by imaging varying cell densities $\left(0-5 \times 10^{5}\right.$ cells/well) of MDA-MB-231-Luc and B16-F10-Luc cells using black wall, clear bottom 24 well plates (Costar, Corning, NY). After incubating the cells for $24 \mathrm{~h}, 400 \mu \mathrm{L}$ of D-luciferin (30 mg/mL; Goldbio, St. Louis, MO) was added to the cells. A dynamic scan for a maximum of up to 40 minutes with $2 \mathrm{sec}$ exposures was acquired (Emission filter: open, f/stop: 1, binning: medium, $1 \mathrm{scan} /$ minute). Pertinent regions of interest (ROI) were drawn on each well and the BLI output was quantified as total flux (photons/second; Living Image software, version 4.2, PerkinElmer Inc., Waltham, MA). To account for any background luminescence, wells without cells (media alone) or wells with cells but lacking the luciferase substrate D-luciferin were included as controls.

For in vivo BLI, animals were injected intraperitoneally with D-luciferin $(300 \mathrm{mg} / \mathrm{kg}$ [0.05 mL/10 g of body weight]) and anesthetized 5 minutes before the peak luciferin uptake time (determined by the in vitro BLI experiment) with 2$3 \%$ isoflurane. Isoflurane was reduced to $2 \%$ after transferring animals to the imaging chamber. Dorsal and ventral BLI (maximum of five animals) was performed (IVIS, PerkinElmer Inc., Waltham, MA) at the optimal imaging time. Weekly in vivo BLI was performed for both models, initiated for the intravenous experimental metastatic model at 3 days after cell implantation and the fat pad spontaneous model was initiated at implantation of cancer cells. Depending on the variability of the bioluminescence signal due to difference in tumor burden, autoexposure (1 second-3 minutes) was used for each cell line/animal. To avoid bleedover from the implantation site, the primary tumor/injection site was covered using a black glove (Dynarex Corporation, Orangeburg, NY) while imaging metastatic lesions in the thoracic cavity. Moreover, bioluminescence tomography or diffuse light imaging tomography (DLIT) on the metastasis model was performed to quantify the number of cancer cells in the lung by utilizing the filtered $2 \mathrm{D}$ bioluminescent sequence (excitation filter: Block, emission filter: 560, 580,
600,620 , and $640 \mathrm{~nm}$ ) in combination with a surface topography.

Determination of the optimal BLI acquisition for the 4T1Luc spontaneous metastasis model was assessed by imaging the primary tumors one week after tumor implantation for a 30-minute dynamic scan (emission filter: open, f/stop: 1, binning: medium, $1 \mathrm{scan} /$ minute, exposure time: $0.5-5$ second). Metastatic burden was evaluated by drawing a standard rectangular shaped ROI (same size over all time points) over the lung region. In addition, $2 \mathrm{D}$ ex vivo BLI was performed on a subset of animals (five weeks after tumor implantation). Prior to necropsy, animals were injected intraperitoneally (i.p.) with D-luciferin $(300 \mathrm{mg} / \mathrm{kg}$ [0.05 mL/10 g of body weight]) and tissues of interest (primary tumor, spleen, liver, lymph nodes, kidneys, femur, heart, brain, adrenal gland, and lung) were resected for ex vivo imaging. After ex vivo imaging, the excised tissues were fixed in 10\% neutral-buffered formalin for histopathological analysis.

2.3. 18-Fluorodeoxyglucose-Positron Emission Tomography/ Computed Tomography Imaging $\left(\left[{ }^{18} F\right] F D G-P E T / C T\right)$. Whole body $\left[{ }^{18}\right.$ F]FDG PET/CT (Inveon Multimodality, Siemens Medical Solutions USA, Inc., Knoxville, TN) was performed to analyze the anatomical location and metabolic function of the lung metastatic tumor [B16-F10-Luc] model 3 weeks after cell implantation. Animals were fasted but allowed water for 10 hours prior to imaging and i.v. tail-vein-injected with $\left[{ }^{18} \mathrm{~F}\right]$ FDG $(7.63 \pm 1.76 \mathrm{MBq}) 60$ minutes prior to imaging. Mice were placed in a heated anesthesia (2\% isoflurane with oxygen carrier at $1 \mathrm{~L} / \mathrm{min}$ flow) induction chamber during the 60-minute uptake period to minimize $\left[{ }^{18} \mathrm{~F}\right] \mathrm{FDG}$ muscle and brown fat uptake. Animal body temperature was maintained before and during imaging sessions using a thermostat controlled circulating warm air imaging table maintained at $37^{\circ} \mathrm{C}$. Pulmonary function was monitored during the imaging sessions and the anesthesia was regulated to maintain a pulmonary rate between 50 and $90 \mathrm{bpm}$. Mice were imaged in the prone position for 5 minutes (CT) followed by 30 minutes (PET) to coregister the metabolic with anatomical image data sets. The following CT acquisition parameters were used: $80 \mathrm{kVp}, 500 \mu \mathrm{A}, 200 \mathrm{msec}$ per step, and 120 steps covering 220 degrees. PET list-mode data was acquired using a $\gamma$-ray energy window of $350-650 \mathrm{keV}$ with a coincidence-timing window of $3.432 \mathrm{~ns}$. CT Cone beam reconstruction resulted in $192 \times 192$ matrix and PET reconstruction utilized OSEM3D, 4 iterations, resulting in a $256 \times 256$ matrix. Images were analyzed using ASIPro software, version 6.8.0.0 (Siemens Medical Solutions USA, Knoxville, TN). Coregistration of the data sets was performed according to manufacturer's recommendations for in-line serial CT-PET scanner. Quantification of lung metastatic tumor uptake (\%ID) was determined with a manual ROI of the total lung subtracted from a $25 \%$ of maximum threshold ROI of the heart. Conversion of counts within an ROI to activity (MBq) was performed according to the manufacturer's procedure.

2.4. T2W-MRI. Representative animals from both tumor models were imaged at week 4 using a $3.0 \mathrm{~T}$ clinical scanner 
(Philips Intera Achieva, Best, The Netherlands) with $40 \mathrm{~mm}$ diameter solenoid receiver volume coil (Philips Research, Hamburg, Germany). A T2-weighted Turbo Spin Echo (T2W-TSE) sequence was implemented (repetition time (TR) of $3500 \mathrm{~ms}$, echo time (TE) of $65 \mathrm{~ms}$, in plane resolution of $0.19 \times 0.19 \mathrm{~mm}^{2}$, and slice thickness of $0.5 \mathrm{~mm}$ ) in the coronal view with respiratory triggering to minimize motion artifacts.

2.5. Histopathology. All animals were euthanized by $\mathrm{CO}_{2}$ asphyxiation either at the end of the study or due to tumor burden associated clinical signs such as greater than $20 \%$ body weight loss, respiratory distress, or paralysis. All mice underwent a thorough necropsy. Tissues were fixed in $10 \%$ neutral-buffered formalin, routinely processed, and embedded in paraffin blocks. For both metastasis models, paraffin blocks of all lungs and tissues noted to have abnormalities at the time of necropsy were sectioned at $5 \mu \mathrm{m}$, stained with hematoxylin and eosin $(\mathrm{H} \& \mathrm{E})$, and microscopically evaluated. Stained sections were scanned into digital format via an Aperio ScanScope XT (Leica, Vista, CA). To calculate areas occupied by metastases, the digital images were annotated by a board-certified veterinary pathologist using Aperio ImageScope software (version 11.2.0.780).

2.5.1. Statistical Analysis. Bioluminescence intensity (photons/second) and tumor measurements were expressed as the mean and standard deviation. Regression analysis (Pearson's correlation method) was performed to demonstrate correlation between luminescence signal intensity, area of the lesion, and lung metastatic lesion $\left[{ }^{18} \mathrm{~F}\right] \mathrm{FDG}$ uptake to histopathology.

\section{Results}

3.1. In Vitro BLI Validation of Luciferase-Expressing Cancer Cells. In vitro evaluation of BLI was performed to determine the efficiency of the bioluminescent signal with respect to cell density (time-to-peak analysis) and sensitivity of signal detection of the cell lines and to correlate cell density with the in vivo signal. Serial dilutions of MDA-MB-231-Luc and B16-F10-Luc cells were plated at different cell densities (0$5 \times 10^{5}$ cells/well) and BLI was performed. An increase in signal was detected with an increase in cell number for both cell lines, suggestive of stability and sustained expression of the luciferase construct (Figure 1). A linear correlation of signal intensity and cell number was obtained for both cell lines in the 3- to 40-minute dynamic range. Based on the dynamic scan, plateau was observed between 22 and 36 minutes, 15 and 30 minutes, and 15 and 22 minutes for B16-F10 cells, MDA-MB-231, and 4T1 cells (data not shown), respectively. These time windows were used as optimal in vivo imaging times for the respective cell lines. As previously reported, signal intensity depends on various factors, such as the stable expression of luciferase (signal intensity per cell), signal detection limitation of the instrument, and attenuation of in vivo signal by the tissues [20]. All cells were maintained up to 20 passages and assayed for in vitro luciferase expression prior to an in vivo study.
3.2. Validation of Metastasis Models by In Vivo BLI. Mice were i.v. tail-vein-injected with MDA-MB-231-Luc $\left(2 \times 10^{6}\right.$ cells $)$ and B16-F10-Luc $\left(1 \times 10^{5}\right.$ cells $)$ cells and monitored by BLI weekly to validate the metastasis model. The lung and other organ BLI signals from the MDA-MB-231-Luc cells were comparable to background (see Figure S1 available online at http://dx.doi.org/10.1155/2014/102702), suggesting rapid clearance of cancer cells from the systemic circulation. The B16-F10-Luc cells successfully colonized the lung as evaluated by increased bioluminescence signal (Figure 2(b)) and histopathological analysis (Figure S2) during the 4-week study. One B16-F10-Luc animal was euthanized 3 weeks after implantation due to excessive tumor burden. In addition, 3D DLIT was performed and demonstrated the presence of luciferase-expressing cancer cells localized around the thoracic region (Figure $2(\mathrm{c})$ ). Bioluminescence quantitation confirmed an increase in signal intensity over the 4-week longitudinal imaging period (Figure 2(d)). DLIT for the MDA-MB-231-Luc model showed relatively fewer cells $(0.31 \%$ cells at four weeks) in the lung (Figure S1C) suggestive of poor colonization of cancer cells.

3.3. Validation of Melanoma Cancer Cell Metastasis by $\left[{ }^{18}\right.$ F]FDG-PET/CT and MRI. The metabolic activity of B16F10-Luc melanoma cells at the metastatic sites was evaluated by the glucose analog PET radiotracer $\left[{ }^{18} \mathrm{~F}\right] \mathrm{FDG}$ and correlated to anatomical locations by MRI. CT was used to correct the $511 \mathrm{keV}$ photon attenuation and to produce images that provided anatomical landmarks. Accumulation of the $\left[{ }^{18} \mathrm{~F}\right]$ FDG was observed in the lung (metastatic lesions) and abdomen and confirmed by coregistration of the PET metabolic image with the anatomical CT (Figure 3) and T2WMRI (Figure 4). As expected, increased [ $\left.{ }^{18} \mathrm{~F}\right] \mathrm{FDG}$ uptake in the heart and bladder was also observed (Figure 4). The uptake of $\left[{ }^{18} \mathrm{~F}\right] \mathrm{FDG}$ correlated with the extent of tumor cell metastasis as evaluated by the H\&E stained section of the lung (Figure 5(a)). By histopathology, there was a metastatic lesion found in the kidney, which was difficult to distinguish from the PET signal due to the renal clearance of the radiotracer (data not shown; Table 1). Regression analysis showed a very good correlation between the percent-injected dose of radiotracer uptake in the lung to percent lung involvement by histopathology and area of lung metastasis to total photon flux (Figures 5(b) and 5(c); Table 1). By histopathology, additional metastatic lesions of melanoma cancer cells were identified in the mediastinum, mediastinal lymph node, and mesentery that did not show accumulation of the radiotracer $\left[{ }^{18} \mathrm{~F}\right]$ FDG (Table 1$)$.

3.4. Mammary Cancer Metastasis and Correlation with T2WMRI and Ex Vivo BLI. Longitudinal BLI was performed for the murine mammary cancer cell line (4T1-Luc) orthotopically implanted in the fourth inguinal mammary fat pad with an intact or resected primary tumor (Figure 6(a)). BLI of both animal groups was performed weekly for five weeks after tumor cell implantation. In one group of animals, the primary tumor was resected once it reached $10 \mathrm{~mm}$ in diameter, as determined by caliper measurements. Successful 


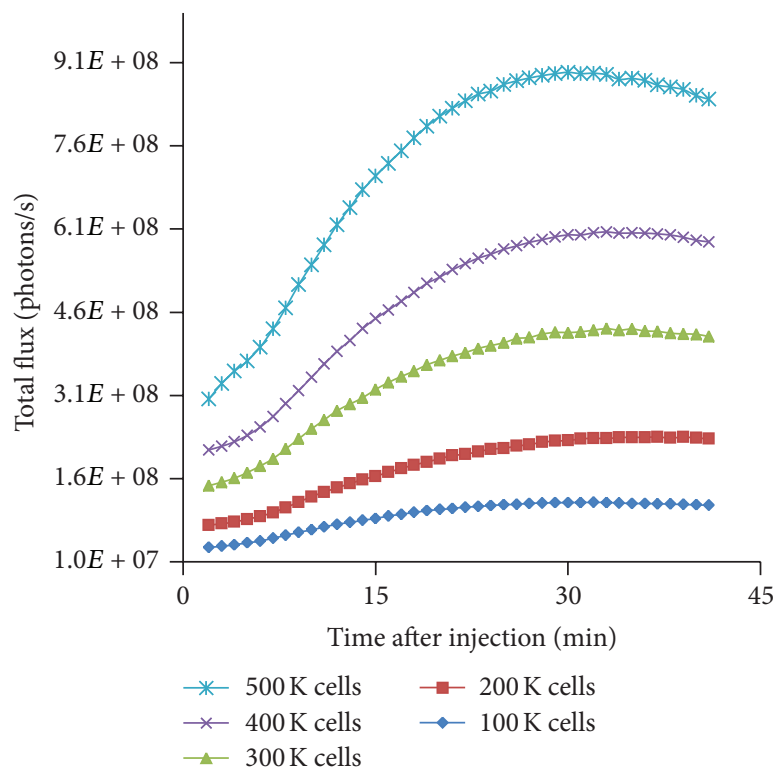

(a)

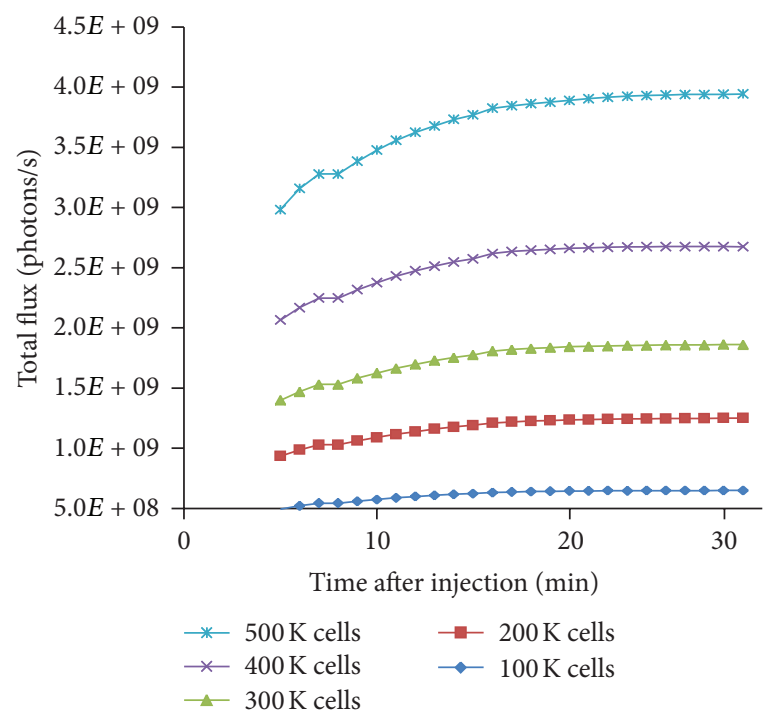

(c)

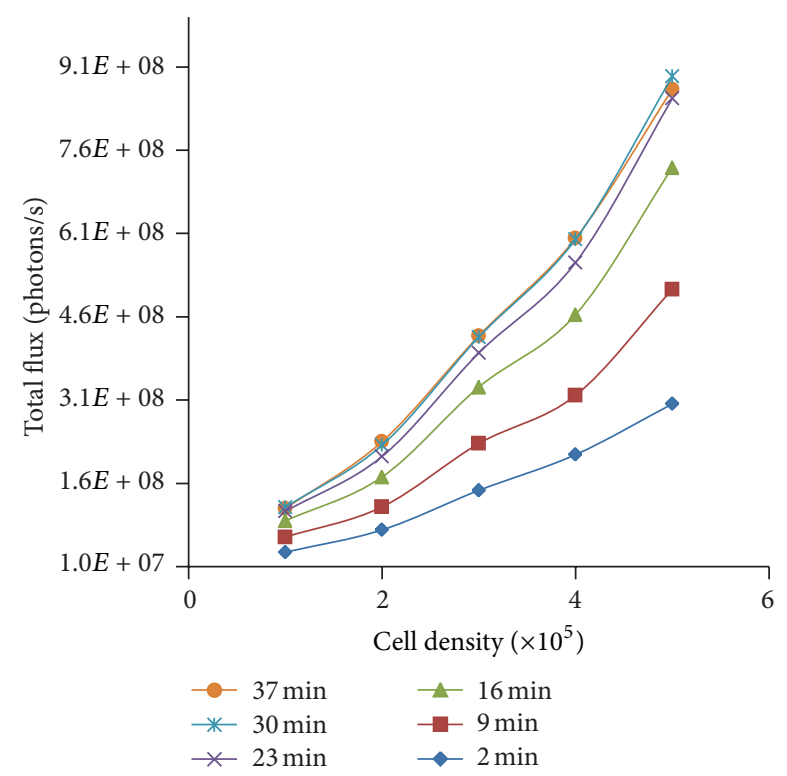

(b)

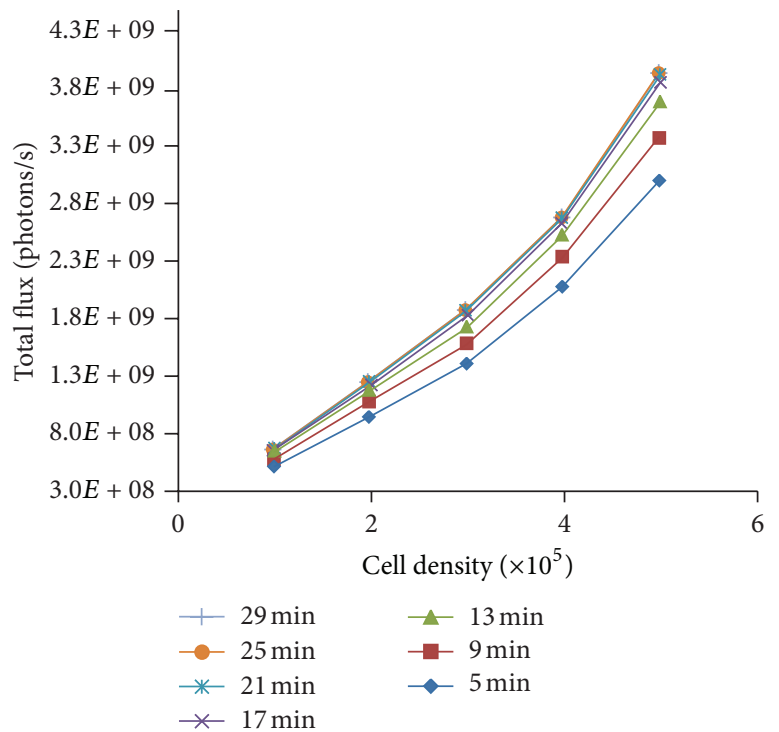

(d)

FIGURE 1: In vitro bioluminescence characteristics of B16-F10-Luc and MDA-MB-231-Luc luciferase-expressing cell lines. Murine melanoma B16-F10-Luc ((a) and (b)) and human breast cancer MDA-MB-231-Luc ((c) and (d)) cell lines were serially diluted and imaged with 2 sec exposures for a maximum of up to 40-minute dynamic scan. Kinetic measurement of luciferase activity correlated with the cell density for both cell lines and luminescence activity increased with time. The assay was done in duplicate.

tumor growth at the primary site was evident by detection of the BLI signal as early as one week after cell implantation (Figure 6(a)). Metastatic spread of the cancer cells to the thoracic/abdominal regions was observed by weeks 2 and 3 by blocking the BLI signal arising from the primary implantation site (Figure S3). Quantitation of the BL signal over time demonstrated in all groups a progressive increase in cancer cell colonization and tumor growth (Figure 6(b)). Tumor regrowth at the primary site following resection was observed in all animals (Figure 6(b)). In addition, there was no significant difference in survival between animals with intact or resected primary tumors (data not shown).
All animals were euthanized by $\mathrm{CO}_{2}$ asphyxiation due to neoplasia-related endpoints.

A biphasic growth pattern was observed with an increased tumor cell proliferation during the first three weeks, and regression after the fourth week, which is indicative of the presence of hypoxic regions or central tumor necrosis. As luciferase enzyme activity requires molecular oxygen in addition to ATP to provide a luminescence signal, this could be a limiting factor in determining the difference between hypoxic and necrotic tissues until the tissue is histologically analyzed. The presence of metastatic lesions as identified by BLI showed association with T2W-MRI (as shown with two 


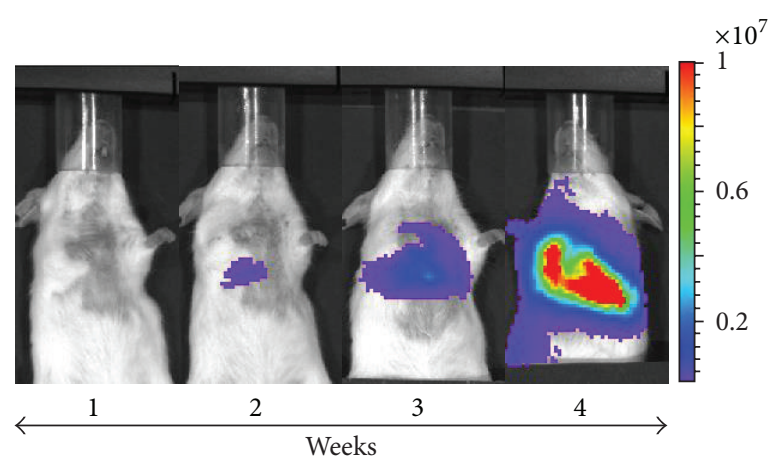

(a)

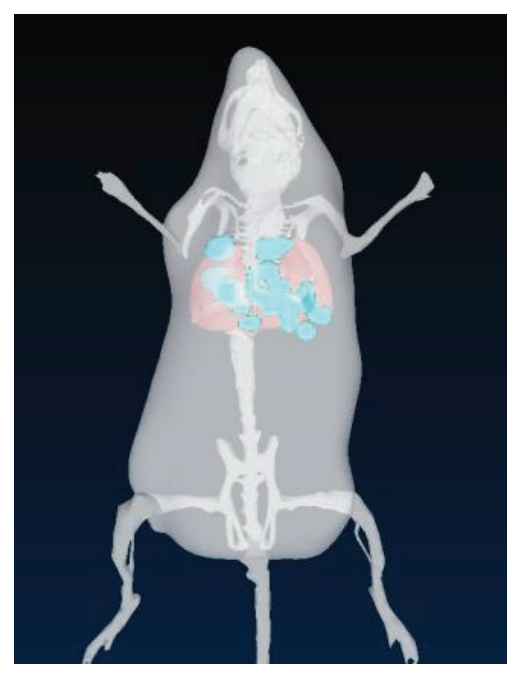

(c)

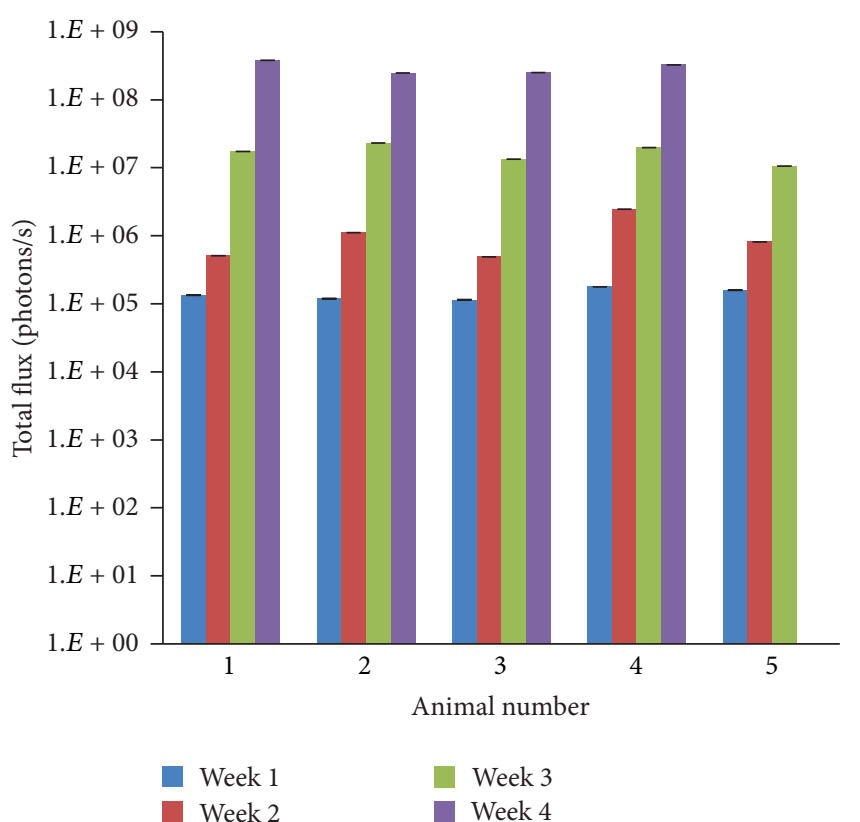

(b)

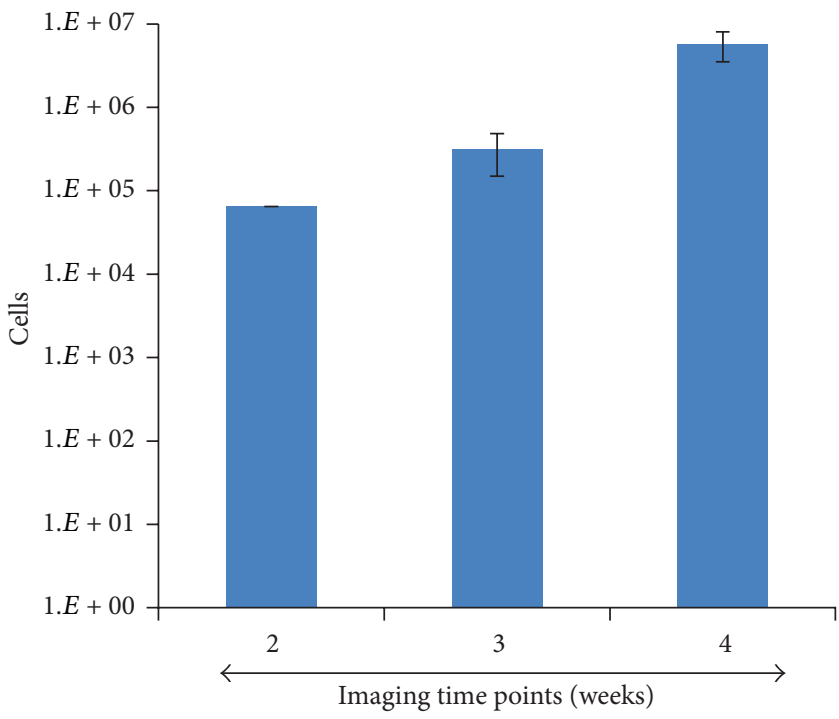

(d)

FIGURE 2: Longitudinal monitoring of tumor growth at metastatic sites by bioluminescence imaging. B16-F10-Luc cells were intravenously injected into female C57 BL $\backslash 6$ mice $(n=5)$. (a) BLI of a representative animal imaged weekly for 4 weeks. (b) Plot representing quantification of bioluminescence intensity as total flux (photons/second) over 4 weeks for all 5 animals. (c) Three-dimensional reconstruction of DLIT of a representative animal at week 4. (d) Quantification of light intensity bioluminescent cells over 3 weeks from DLIT reconstruction.

different slices) at the left cervical lymph node and lung (Figure 6(c)). Ex vivo examination by BLI of various organs further confirmed the presence and extent of cancer cell infiltration in the lung and left cervical lymph node.

\subsection{Correlation of In Vivo Imaging with Histopathology.} Histopathological analysis was performed on both metastatic models to determine the presence of tumor cell infiltration in various organs and correlation with BLI. At the end of the study (4 weeks for B16-F10-Luc and 5 weeks for 4T1-Luc cells), animals were euthanized and the presence of lung metastasis was confirmed in all animals (100\%) with less frequent metastases noted in other organs. The extent of tumor cells in the lung for both metastatic models varied between animals but showed good correlation $\left(R^{2}=0.74\right.$ for B16-F10-Luc cells and $R^{2}=0.7$ for 4T1-Luc cells) with the signal intensity obtained from BLI (Figures 5(c) and 6(e)).

3.6. Histopathology Findings. Histopathological analysis identified additional organs (sites) that contained tumor cells 


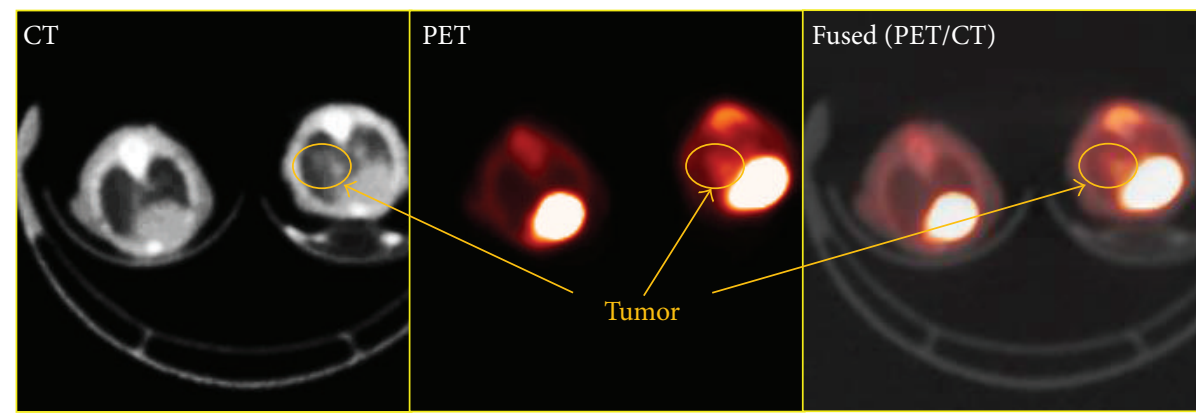

FIGURE 3: Imaging melanoma cancer cell metastasis by $\left[{ }^{18} \mathrm{~F}\right] \mathrm{FDG}-\mathrm{PET} / \mathrm{CT}$. Evaluation of fused PET/CT images confirms an increase in $\left[{ }^{18} \mathrm{~F}\right] \mathrm{FDG}$ accumulation in the lungs of animals with metastatic lesions as shown on transverse slices. Images were obtained 21 days after cancer cell injection.

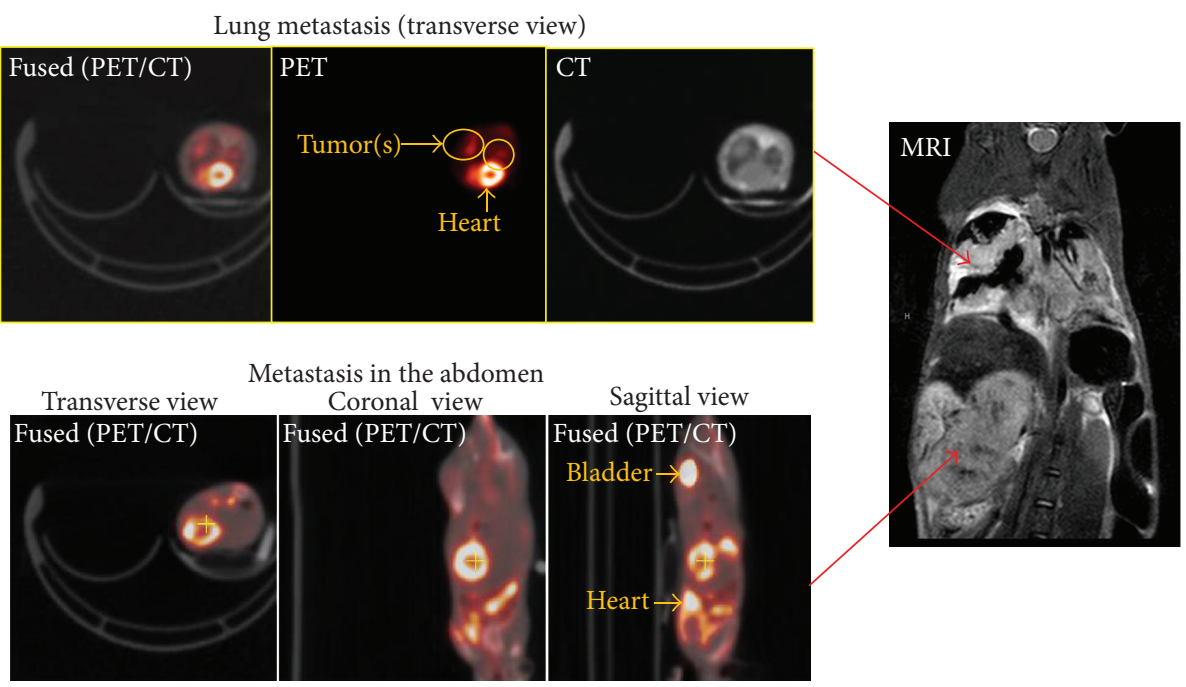

FIGURE 4: A correlation of $\left[{ }^{18} \mathrm{~F}\right] \mathrm{FDG}-\mathrm{PET} / \mathrm{CT}$ and T2W-MRI of melanoma cancer cell metastasis. Accumulation of $\left[{ }^{18} \mathrm{~F}\right] \mathrm{FDG}$ in the lungs and abdomen as evaluated by fused PET/CT correlated with metastatic lesions observed by T2W-MRI. A representative image from five animals is shown.

in both metastatic models. These findings are summarized in Table 2. Histopathology also confirmed the presence of cancer cells in the adrenal, mediastinum, brain, kidney, liver, thymus, vertebra, and heart for the spontaneous metastatic model with an intact primary tumor. Animals that had the primary tumor resected displayed metastases in the mediastinum, thymus, rib, and adrenal at a lower frequency than the nonresected model.

\section{Discussion}

4.1. Noninvasive Imaging of Tumor Growth at Metastatic Sites. The present study provides a method to characterize two syngeneic metastatic models (C57 BL/6 and Balb/c mouse strains) using multiple imaging modalities to detect secondary tumors and validate these findings with histopathology. Both metastatic models were validated using murine cancer cell lines that stably overexpress the luciferase gene at a high level as evaluated by in vitro BLI. The BLI signal intensity correlated with the number of cells in an in vitro assay demonstrating the ease in monitoring and quantifying proliferation of cancer cells. Tumor growth of B16-F10-Luc cells in the experimental metastasis model and 4T1-Luc cells implanted orthotopically in the mammary fat pad for the spontaneous metastasis model resulted in colonization of cancer cells primarily in the lung. Metastasis observed using syngeneic cancer cells showed similarities to metastasis and dissemination to organs as seen in clinical settings [24]. Immunocompetent animals have an intact immune system that plays a critical role in the progression and metastasis of cancer cells. For instance, tumor growth and metastasis of $4 \mathrm{~T} 1$ cells in Balb/c animals are influenced by both innate and adaptive immune responses [25].

Longitudinal BLI confirmed the progression of tumor growth at metastatic sites in both tumor models. Similar to a previous report, we observed fewer lung metastases with MDA-MB-231-Luc cells when injected systemically through the lateral tail-vein $[22,26,27]$. Differences in tumor take rate and metastasis can be attributed to the use of different clonal cells and/or mouse strains (e.g., nude and nudebeige mice [22]). Progressive tumor growth was observed with an increase in signal intensity over 4-5 weeks for both 

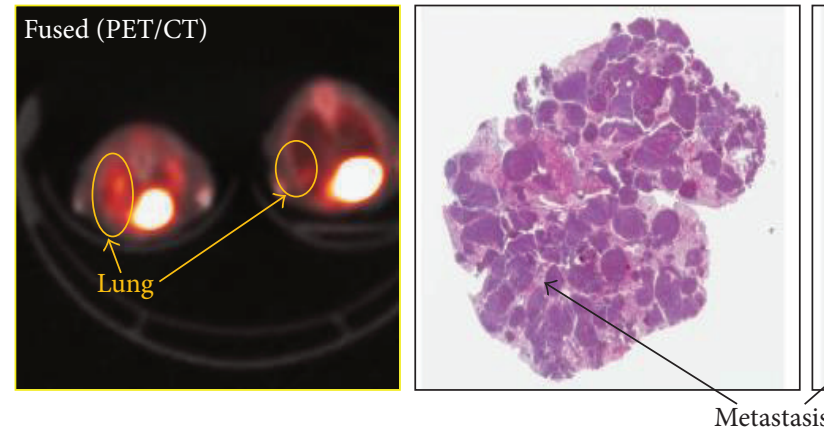

(a)

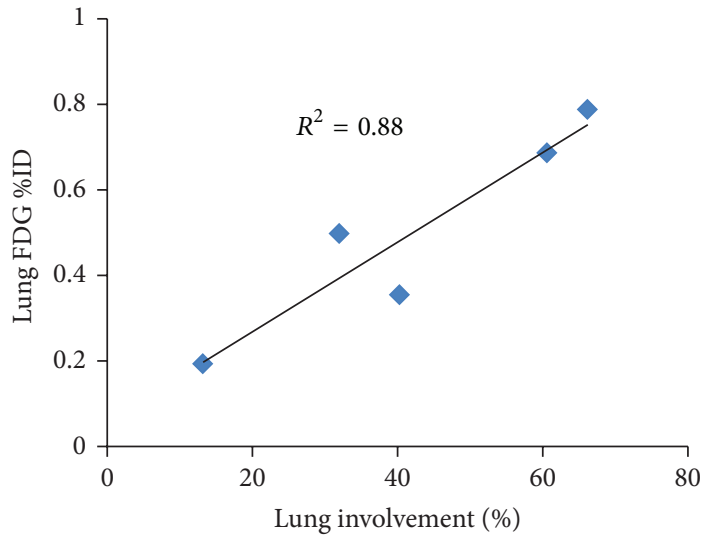

(b)

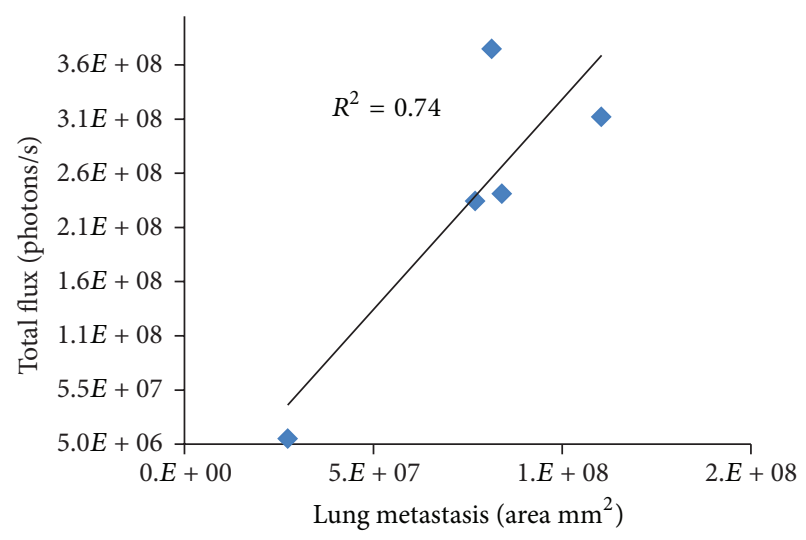

(c)

FIGURE 5: Correlation of $\left[{ }^{18} \mathrm{~F}\right]$ FDG-PET imaging of melanoma cancer cell metastasis with histopathology. $\left[{ }^{18} \mathrm{~F}\right] \mathrm{FDG}$ accumulation in the lungs correlated with the extent and presence of metastasis by histopathology. (a) Representative fused image of PET/CT from 2 animals showing different levels of $\left[{ }^{18} \mathrm{~F}\right] \mathrm{FDG}$ accumulation. Correlation with tumor nodules in the lung is evident from paraffin section images of the lung stained with H\&E. (b) Regression plot showing correlation between lung metastatic lesions identified by $\left[{ }^{18}\right.$ F]FDG-PET imaging and the percent of lung involvement of melanoma cancer cells calculated by histopathology. (c) Regression plot between BLI signal intensity of the thoracic region and the lung metastasis area $\left(\mathrm{mm}^{2}\right)$ as calculated by histopathology. Correlation was calculated using Pearson correlation coefficient analysis.

tumor models (B16-F10-Luc and 4T1-Luc cells), confirming successful colonization of cancer cells at metastatic sites such as the thoracic region. Using DLIT for the B16-F10-Luc model, we were able to obtain tomographic images of tumor locations based on signal intensity, which was suggestive of lung metastasis. This same technique was employed to reconstruct a $3 \mathrm{D}$ distribution of intracranially implanted murine glioma cells in the brain region [19]. In addition to BLI, tumor growth in the lung was confirmed by $\left[{ }^{18} \mathrm{~F}\right] \mathrm{FDG}$ PET to image metabolic function of the tumor and coregister with anatomical X-ray CT and T2W-MRI. In addition to lung metastasis, correlation of metastasis in the abdomen was observed by $\left[{ }^{18} \mathrm{~F}\right] \mathrm{FDG}$ PET/CT and T2W-MRI. In an independent study, a similar detection of metastatic lesions in the lung and abdomen was observed with fused CT/PET imaging for the B16 melanoma subline [28]. Similarly, a very high correlation of the mean uptake of PET radiotracer within the lung metastasis was observed in 4 of 5 SCID animals intravenously injected with A375 M-Fluc melanoma cells [29]. Several tumor models have been validated using small animal PET, BLI, MRI, and micro-CT imaging modalities as they provide complementary information [5, 28, 29]. For instance, fusion of small animal PET and micro-CT imaging correlated with metabolic function to the anatomical location of lung tumors [28]. In several reports, systemic administration by tail-vein of different cancer cell lines has resulted in the induction of lung tumors [28, 30], with the exception of the mouse pheochromocytoma luciferaseexpressing cell line, which results in liver metastasis [14].

Differences in the route and density of cancer cell injection have resulted in metastatic lesions in various organs [3133]. For example, intracardiac injection of $10^{2} \mathrm{~B} 16$ melanoma cells resulted in bone metastasis, but systemic administration of the same number of cells by i.v. tail-vein did not result in lung metastasis [31]. In contrast, increasing the number of cells to $10^{5}$ by i.v. tail-vein injection resulted in lung metastasis with no apparent bone lesions. In another study, subcutaneous administration of B16 melanoma cancer cells into the ear pinna resulted in cervical lymph node metastasis with no evidence of distant spread of cancer cells [32]. In 
TABLE 1: Summary of melanoma cancer cell metastases detected by histopathology and $\left[{ }^{18} \mathrm{~F}\right] \mathrm{FDG}-\mathrm{PET}$.

\begin{tabular}{|c|c|c|c|c|c|c|}
\hline \multirow[b]{2}{*}{$\begin{array}{l}\text { Tissues with melanoma } \\
\text { cancer cell metastasis }\end{array}$} & \multirow[b]{2}{*}{ Method of detection } & \multicolumn{5}{|c|}{ Mouse number } \\
\hline & & 1 & 2 & 3 & 4 & 5 \\
\hline \multirow{2}{*}{ Lung } & {$\left[{ }^{18} \mathrm{~F}\right] \mathrm{FDG}-\mathrm{PET}$} & + & + & + & + & + \\
\hline & Histopathology & + & + & + & + & + \\
\hline \multirow{2}{*}{ Mediastinum } & {$\left[{ }^{18} \mathrm{~F}\right]$ FDG-PET } & - & - & - & - & - \\
\hline & Histopathology & + & - & - & - & + \\
\hline \multirow{2}{*}{ Mediastinal lymph node } & {$\left[{ }^{18} \mathrm{~F}\right]$ FDG-PET } & - & - & - & - & - \\
\hline & Histopathology & - & - & + & + & - \\
\hline \multirow{2}{*}{ Kidney* } & {$\left[{ }^{18} \mathrm{~F}\right]$ FDG-PET } & + & + & + & + & + \\
\hline & Histopathology & - & + & - & - & + \\
\hline \multirow{2}{*}{ Mesentery } & {$\left[{ }^{18} \mathrm{~F}\right]$ FDG-PET } & - & - & - & - & - \\
\hline & Histopathology & - & - & - & - & + \\
\hline $\begin{array}{l}\text { Percentage of lung } \\
\text { section involved in } \\
\text { melanoma cancer cell } \\
\text { metastasis }\end{array}$ & & 40.3 & 13.2 & 32.0 & 66.2 & 60.6 \\
\hline $\begin{array}{l}\text { Percent injected dose of } \\
{\left[{ }^{18} \mathrm{~F}\right] \text { FDG in the lung }}\end{array}$ & & 0.35 & 0.19 & 0.49 & 0.78 & 0.69 \\
\hline
\end{tabular}

+: metastasis; -: no metastasis; ${ }^{*}\left[{ }^{18} \mathrm{~F}\right] \mathrm{FDG}-\mathrm{PET}$ is cleared primarily through kidney and may not be a true reflection of presence of metastasis.

TABLE 2: Comparison of organs with tumor lesions detected by histopathology between the two metastatic models.

\begin{tabular}{|c|c|c|c|}
\hline \multirow{2}{*}{ Organs } & \multirow{2}{*}{$\begin{array}{l}\text { Experimental metastasis (tail-vein injection) } \\
\text { B16-F10 }\end{array}$} & \multicolumn{2}{|c|}{ Spontaneous metastasis (mammary fat pad injection) } \\
\hline & & 4T1 (primary tumor resected) & 4T1 (primary tumor intact) \\
\hline Lung & $10 / 10(100 \%)$ & $5 / 5(100 \%)$ & $5 / 5(100 \%)$ \\
\hline Mediastinal lymph node & $4 / 10(40 \%)$ & - & - \\
\hline Mediastinum & $5 / 10(50 \%)$ & $2 / 5(40 \%)$ & $3 / 5(60 \%)$ \\
\hline Vertebra/spinal cord & $2 / 10(20 \%)$ & - & $1 / 5(20 \%)$ \\
\hline Brain & - & - & $2 / 5(40 \%)$ \\
\hline Kidney & $2 / 10(20 \%)$ & - & $1 / 5(20 \%)$ \\
\hline Liver & - & - & $2 / 5(40 \%)$ \\
\hline Thymus & - & $2 / 5(40 \%)$ & $2 / 5(40 \%)$ \\
\hline Rib & - & $2 / 5(40 \%)$ & - \\
\hline Adrenal & - & $1 / 5(20 \%)$ & $4 / 5(80 \%)$ \\
\hline Heart & - & - & $1 / 5(20 \%)$ \\
\hline Mesentery & $1 / 10(10 \%)$ & - & - \\
\hline Subcutaneous lymph node & - & $2 / 5(40 \%)$ & $1 / 5(20 \%)$ \\
\hline Sternum and foreleg & - & - & $1 / 5(20 \%)$ \\
\hline
\end{tabular}

addition to the injection route, isolation of cancer cells from a particular organ can significantly bias the dissemination of metastatic lesions to that organ. For example, isolation of brain seeking breast cancer cells (MDA-MB-231 BR) shows preferential metastasis to the brain when cells are injected via an intracardiac route [34]. Similarly, isolation of the MDAMB-231 D3H2LN subclone showed an increased tumor take rate and spontaneous lymph node metastasis compared to the parental D3H1 subclone cells [22]. Furthermore, there have been discrepancies in the observed metastasis by cancer cells. In one study, intravenous injection of A549-Luc resulted in no detectable metastases [35] while another report showed successful cancer cell colonization in the lung [30]. Differences observed in colonization of cancer cells and detection sensitivity can be attributed to differences in the luciferase construct, the number of cancer cells injected, the injection needle gauge, and other study-related variables.

The tumorigenic potential of cancer cells can also vary depending on the isolation procedure, as observed for colorectal cancer cells, LS174T [33]. Differences in metastatic frequency were observed by intraportal injection with LS174T cells harvested from a culture plate with or without the use of enzymatic trypsin (isolated single cells versus aggregated cells). The presence of intact surface adhesion markers in 


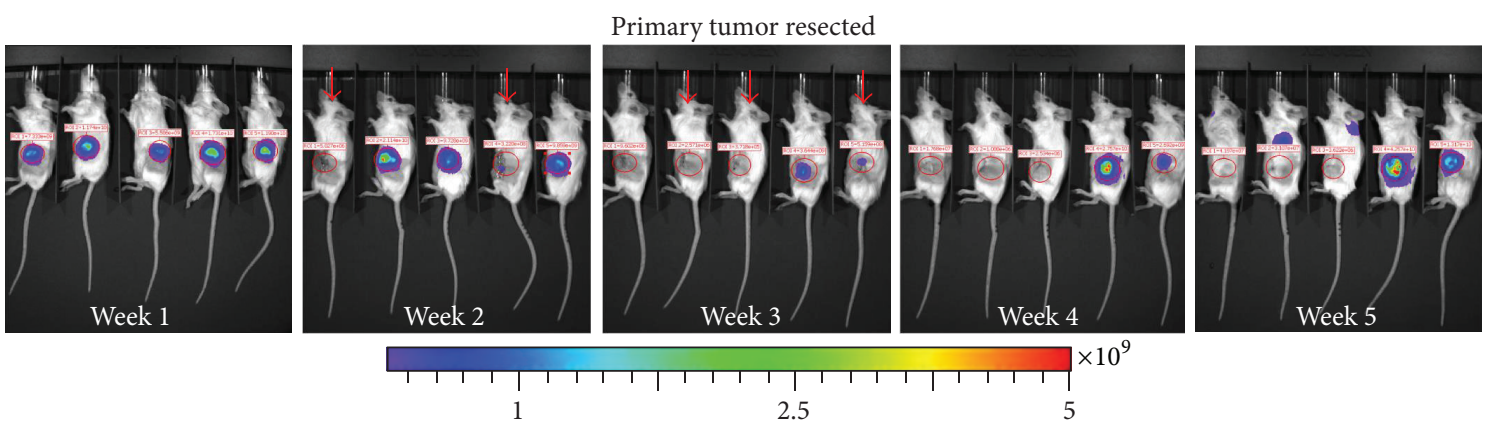

Primary tumor intact
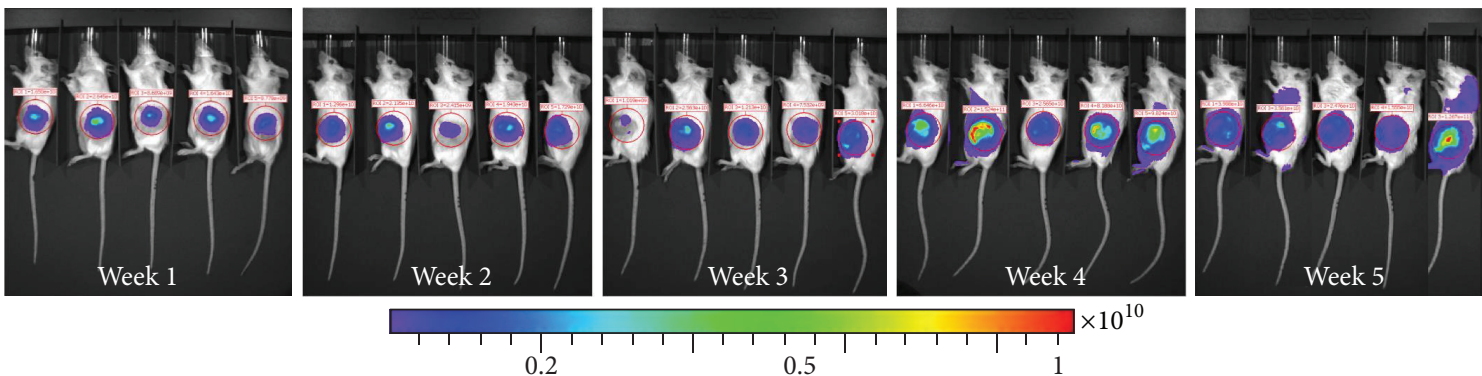

(a)
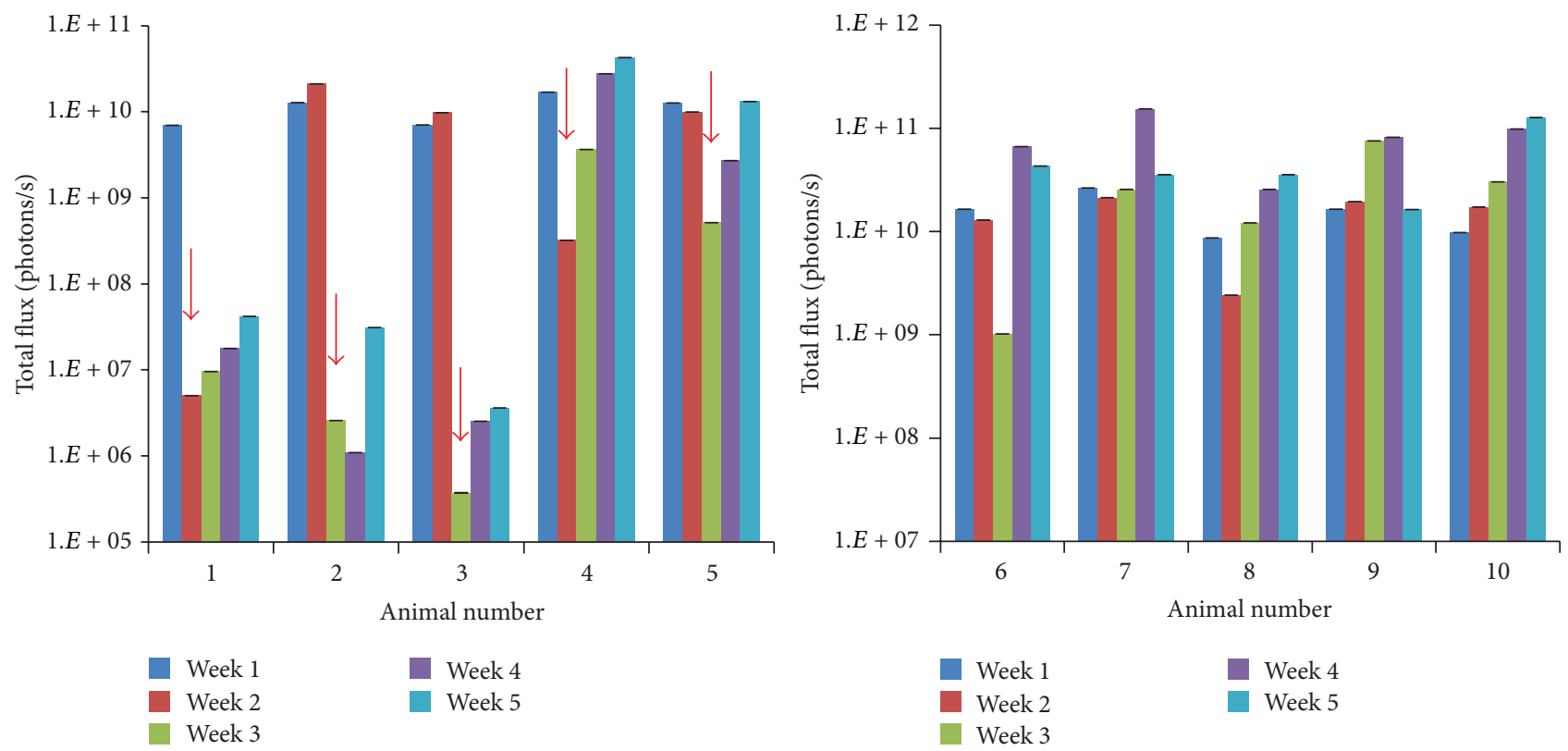

(b)

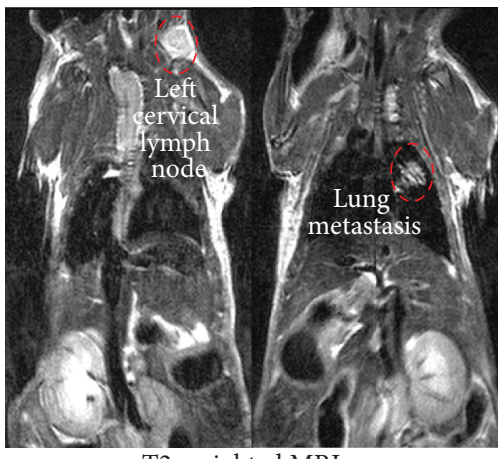

T2-weighted MRI

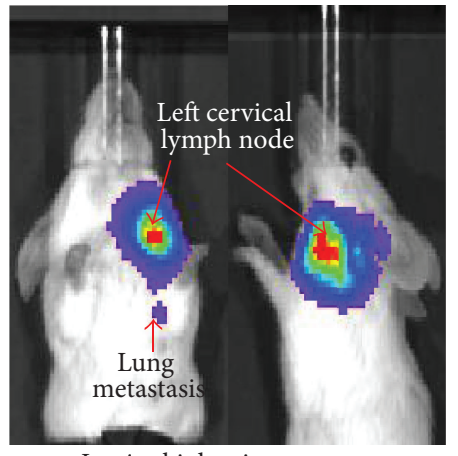

In-vivo bioluminescence

(c)

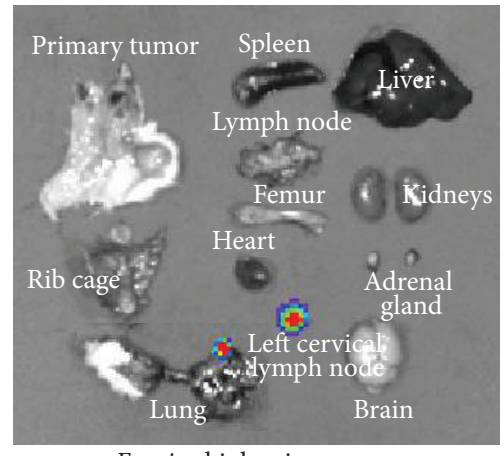

Ex-vivo bioluminescence

Figure 6: Continued. 

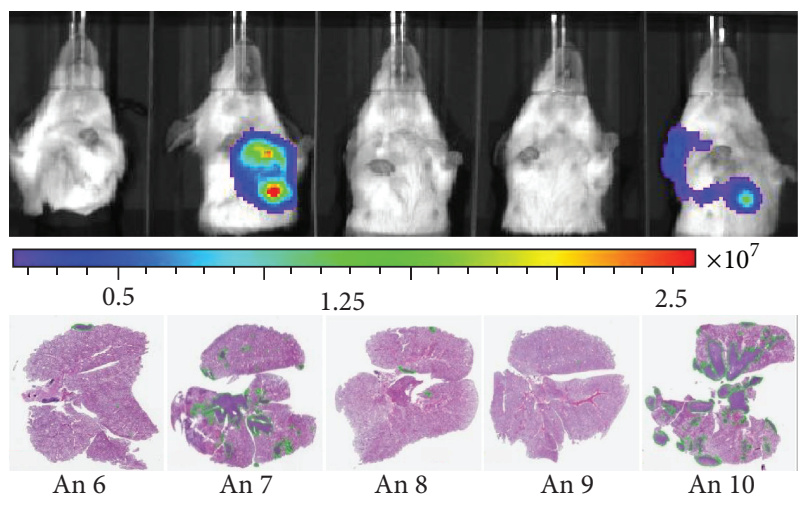

(d)

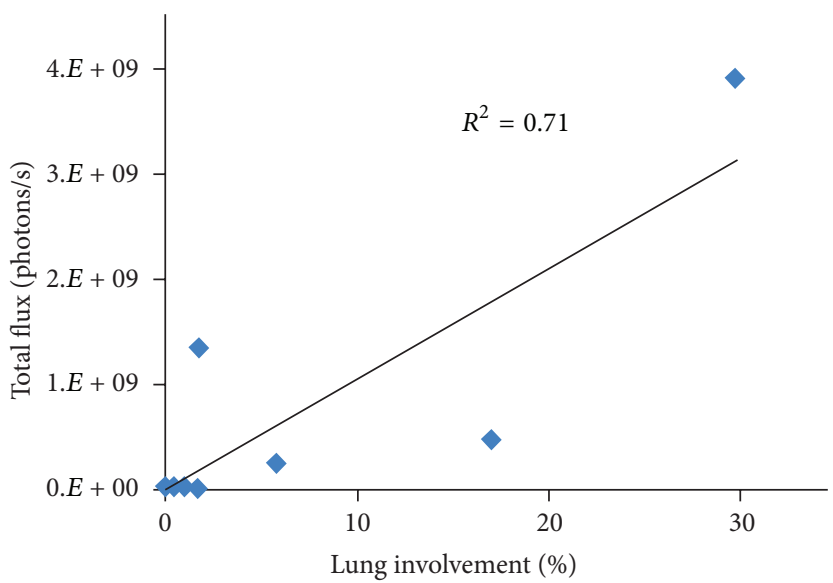

(e)

FIGURE 6: Longitudinal bioluminescence imaging of mammary cancer cell metastasis in a syngeneic mouse model and correlation with histopathology. (a) 4T1-Luc cells, a murine mammary cancer cell line, were orthotopically injected in the mammary fat pad and serial bioluminescence imaging performed 7, 14, 21, 28, and 35 days after cancer cell implantation is shown. Red arrows indicate points at which primary tumors were resected when reaching $10 \mathrm{~mm}$ in diameter (top graph). In another group of 5 animals, the primary tumors remained intact (bottom graph). (b) BLI quantification of tumor growth at the implantation site (primary tumor) for both groups; the increase in BLI total signal intensity over time suggests progression of cancer at the primary tumor site. (c) BLI signal intensity at metastatic sites was correlated to T2W-MRI and with ex vivo imaging. (d) The correlation of BLI signals with H\&E stained lung sections is shown for all animals with intact primary tumors. The green margins around the tumor lesions in H\&E stained sections were used to calculate the area or percentage of tumor involvement. (e) The plot shows the correlation between the BLI signals at week 5, with percent tumor involvement in the lung calculated by histopathology.

aggregated cells is important for successful colonization in the liver and is the suggested reason for the difference in metastasis between aggregated and isolated single cells.

\subsection{Noninvasive Imaging of Metastasis Correlated with} Histopathology. Noninvasive BLI monitored the extent of metastasis of cancer cells in both models and correlated with histopathology. The first metastasis model involving an intravenous injection of melanoma cancer cells, B16-F10-Luc cells, demonstrated successful colonization of cancer cells in the thoracic region based on BLI. The presence of metabolic lung metastases was confirmed in all animals $(n=5)$ by coregistration of small animal $\left[{ }^{18} \mathrm{~F}\right] \mathrm{FDG}$ PET imaging with small animal X-ray CT demonstrating the anatomical and metabolic details of the cancerous cells. Furthermore, the extent of metastasis in the lung strongly correlated with the mean percent of injected dose uptake of the $\left[{ }^{18} \mathrm{~F}\right] \mathrm{FDG}$ radiotracer in the lung nodules and histology. In addition, T2WMRI detected lung metastasis as previously confirmed by BLI and PET/CT imaging modalities. Surprisingly, in an independent study the formation of lung metastases by intravenous injection of B16-F10 cells was undetected by MRI, which was evident by PET/CT imaging [28]. Due to the excretion and kinetics of $\left[{ }^{18} \mathrm{~F}\right] \mathrm{FDG}$, the observed high $\left[{ }^{18} \mathrm{~F}\right] \mathrm{FDG}$ uptake in both the kidney and heart made it difficult to differentiate the presence of metastatic lesions from preferential uptake of the radiotracer in these organs, as evidenced by histopathology detection of metastatic lesions in the kidney, in addition to the mediastinum and mediastinum lymph nodes.

In our spontaneous metastasis model using 4T1-Luc cells, we detected metastasis in the thoracic region by BLI and by histopathology in all animals both with and without resection of the primary tumor. The extent of cancer cell infiltration in the lung determined by histopathological analysis correlated with the BLI signal. In addition, lesions identified by BLI correlated with the presence of metastasis in the left cervical lymph node as observed by T2W-MRI and ex vivo BLI.

Longitudinal imaging of tumor growth using the BLI technique offers several advantages such as enhanced speed, ease of image acquisition, high throughput, and low cost of operation, making it an attractive noninvasive imaging modality for preclinical models. Histopathology is considered the gold standard in metastases assessment; however, noninvasive imaging provides real-time tumor growth information. BLI has several limitations: signal intensity is dependent on depth (lesions on the surface have higher signal intensity compared to lesions in the deep tissue) and the 2D planar image does not provide the ability to determine lesion depth. Despite these limitations, noninvasive imaging when coupled with histopathology provides a strong correlation for detection of metastatic lesions.

This study provided methods to characterize two metastatic models using multimodal imaging techniques, BLI, $\mathrm{PET} / \mathrm{CT}$, and T2W-MRI, with validation of tumor lesions with histopathology. Based on this study, BLI provides a valid noninvasive imaging strategy to monitor tumor growth in longitudinal studies. Using complementary imaging modalities can circumvent inherent limitations of optical imaging such as signal attenuation due to the tissue depth, availability of luciferin substrate, and low levels of ATP and oxygen at the tumor site. Furthermore, application of other imaging modalities and molecular probes such as small animal PET/CT and 
MRI to image metastatic sites will afford more information about the tumor model (metabolic activity, anatomical location, and tumor volume) and can assist in employing relevant therapeutic intervention strategies. Further validation of metastatic sites with histopathological analysis alongside noninvasive imaging modalities will be helpful in evaluating tumor growth and effectiveness of therapy.

\section{Conclusion}

Overall, we were able to validate two syngeneic metastatic models using various imaging technologies to understand the tumor growth profiles at secondary sites noninvasively and longitudinally. BLI provides a sensitive technique for imaging the whole body, for detection of metastatic lesions, and correlation with T2W-MRI and histopathological analysis. Metabolic $\left[{ }^{18} \mathrm{~F}\right]$ FDG PET/CT of the B16-F10 metastatic model further confirmed the presence of tumors detected by BLI. Each of the imaging modalities provides a specific advantage: small animal PET provides metabolic activity; micro-CT contributes anatomical information of the lung; and T2W-MRI provides morphological and tumor volume data. Based on the present study, it is advantageous to utilize complementary imaging modalities with correlation to histopathology to maximize the understanding of a tumor model and/or response to a therapeutic treatment.

\section{Conflict of Interests}

The authors declare that there is no conflict of interests regarding the publication of this paper.

\section{Acknowledgments}

The authors wish to thank Rachael M. Crist, Frederick National Laboratory for Cancer Research, for assistance with the preparation of the paper. In addition, authors wish to thank Tim Potter for cell culture, Daniel Logsdon for animal handling, and Gloryvee Rivera, Wendi Custer Lawrence, and Kelly Benauer for assistance with histopathology, all from Frederick National Laboratory for Cancer Research. This project was funded in whole or in part by federal funds from the National Cancer Institute, National Institutes of Health, under Contract no. HHSN261200800001E. The content of this paper does not necessarily reflect the views or policies of the Department of Health and Human Services, nor does it mention trade names, commercial products, or organizations that imply endorsement by the U.S. Government.

\section{References}

[1] C. L. Chaffer and R. A. Weinberg, "A perspective on cancer cell metastasis," Science, vol. 331, no. 6024, pp. 1559-1564, 2011.

[2] I. J. Fidler, "Critical determinants of metastasis," Seminars in Cancer Biology, vol. 12, no. 2, pp. 89-96, 2002.

[3] G. Damia and M. D’Incalci, "Contemporary pre-clinical development of anticancer agents-what are the optimal preclinical models?" European Journal of Cancer, vol. 45, no. 16, pp. 27682781, 2009.
[4] B. A. Hoff, K. Chughtai, Y. H. Jeon et al., "Multimodality imaging of tumor and bone response in a mouse model of bony metastasis," Translational Oncology, vol. 5, no. 6, pp. 415-421, 2012.

[5] A. L. Puaux, L. C. Ong, Y. Jin et al., "A comparison of imaging techniques to monitor tumor growth and cancer progression in living animals," International Journal of Molecular Imaging, vol. 2011, Article ID 321538, 12 pages, 2011.

[6] G. van der Horst, J. J. van Asten, A. Figdor et al., "Real-time cancer cell tracking by bioluminescence in a preclinical model of human bladder cancer growth and metastasis," European Urology, vol. 60, no. 2, pp. 337-343, 2011.

[7] E. O. Aboagye, F. J. Gilbert, I. N. Fleming et al., "Recommendations for measurement of tumour vascularity with positron emission tomography in early phase clinical trials," European Radiology, vol. 22, no. 7, pp. 1465-1478, 2012.

[8] H. C. Kang, K. S. Tan, S. M. Keefe et al., "MRI assessment of early tumor response in metastatic renal cell carcinoma patients treated with sorafenib," American Journal of Roentgenology, vol. 200, no. 1, pp. 120-126, 2013.

[9] A. Balducci, Y. Wen, Y. Zhang et al., "A novel probe for the non-invasive detection of tumor-associated inflammation," Oncoimmunology, vol. 2, no. 2, Article ID e23034, 2013.

[10] K. Changani, C. Pereira, S. Young et al., "Longitudinal characterisation of a model of chronic allergic lung inflammation in mice using imaging, functional and immunological methods," Journal of Inflammation, vol. 10, supplement 1, p. P4, 2013.

[11] J. P. Holland, P. Cumming, and N. Vasdev, "PET of signal transduction pathways in cancer," Journal of Nuclear Medicine, vol. 53, no. 9, pp. 1333-1336, 2012.

[12] N. Matusiak, A. Waarde, R. Bischoff et al., "Probes for noninvasive matrix metalloproteinase-targeted imaging with PET and SPECT," Current Pharmaceutical Design, vol. 19, no. 25, pp. 4647-4672, 2013.

[13] P. C. Black, A. Shetty, G. A. Brown et al., "Validating bladder cancer xenograft bioluminescence with magnetic resonance imaging: the significance of hypoxia and necrosis," BJU International, vol. 106, no. 11, pp. 1799-1804, 2010.

[14] A. Giubellino, G. M. Woldemichael, C. Sourbier et al., "Characterization of two mouse models of metastatic pheochromocytoma using bioluminescence imaging," Cancer Letters, vol. 316, no. 1, pp. 46-52, 2012.

[15] M. Zabala, P. Alzuguren, C. Benavides et al., "Evaluation of bioluminescent imaging for noninvasive monitoring of colorectal cancer progression in the liver and its response to immunogene therapy," Molecular Cancer, vol. 8, article 2, 2009.

[16] X. Ma, Z. Liu, X. Yang et al., "Dual-modality monitoring of tumor response to cyclophosphamide therapy in mice with bioluminescence imaging and small-animal positron emission tomography," Molecular Imaging, vol. 10, no. 4, pp. 278-283, 2011.

[17] A. Rehemtulla, L. D. Stegman, S. J. Cardozo et al., "Rapid and quantitative assessment of cancer treatment response using in vivo bioluminescence imaging," Neoplasia, vol. 2, no. 6, pp. 491495, 2000.

[18] O. Szentirmai, C. H. Baker, N. Lin et al., "Noninvasive bioluminescence imaging of luciferase expressing intracranial U87 xenografts: correlation with magnetic resonance imaging determined tumor volume and longitudinal use in assessing tumor growth and antiangiogenic treatment effect," Neurosurgery, vol. 58, no. 2, pp. 365-372, 2006. 
[19] M. G. Abdelwahab, T. Sankar, M. C. Preul, and A. C. Scheck, "Intracranial implantation with subsequent $3 \mathrm{D}$ in vivo bioluminescent imaging of murine gliomas," Journal of Visualized Experiments, no. 57, Article ID e3403, 2011.

[20] X. Ji, L. Cheng, F. Wei et al., "Noninvasive visualization of retinoblastoma growth and metastasis via bioluminescence imaging," Investigative Ophthalmology and Visual Science, vol. 50, no. 12, pp. 5544-5551, 2009.

[21] W. W. Overwijk and N. P. Restifo, "B16 as a mouse model for human melanoma," Current Protocols in Immunology, vol. 20, p. 20.1, 2001.

[22] D. E. Jenkins, Y. S. Hornig, Y. Oei, J. Dusich, and T. Purchio, "Bioluminescent human breast cancer cell lines that permit rapid and sensitive in vivo detection of mammary tumors and multiple metastases in immune deficient mice," Breast Cancer Research, vol. 7, no. 4, pp. R444-R454, 2005.

[23] J.-B. Kim, K. Urban, E. Cochran et al., "Non-invasive detection of a small number of bioluminescent cancer cells in vivo," PLoS ONE, vol. 5, no. 2, Article ID e9364, 2010.

[24] A. E. Vernon, S. J. Bakewell, and L. A. Chodosh, "Deciphering the molecular basis of breast cancer metastasis with mouse models," Reviews in Endocrine and Metabolic Disorders, vol. 8, no. 3, pp. 199-213, 2007.

[25] K. Tao, M. Fang, J. Alroy, and G. G. Gary, "Imagable 4T1 model for the study of late stage breast cancer," BMC Cancer, vol. 8, article 228, 2008.

[26] J. E. Price, A. Polyzos, R. D. Zhang, and L. M. Daniels, "Tumorigenicity and metastatis of human breast carcinoma cell lines in nude mice," Cancer Research, vol. 50, no. 3, pp. 717-721, 1990.

[27] K. Wang, S. Xie, Y. Ren, H. Xia, X. Zhang, and J. He, "Establishment of a bioluminescent MDA-MB-231 cell line for human triple-negative breast cancer research," Oncology Reports, vol. 27, no. 6, pp. 1981-1989, 2012.

[28] C. T. Winkelmann, S. D. Figueroa, T. L. Rold, W. A. Volkert, and T. J. Hoffman, "Microimaging characterization of a B16-F10 melanoma metastasis mouse model," Molecular Imaging, vol. 5, no. 2, pp. 105-114, 2006.

[29] C. M. Deroose, A. De, A. M. Loening et al., "Multimodality imaging of tumor xenografts and metastases in mice with combined small-animal PET, small-animal CT, and bioluminescence imaging," Journal of Nuclear Medicine, vol. 48, no. 2, pp. 295-303, 2007.

[30] D. E. Jenkins, Y. Oei, Y. S. Hornig et al., "Bioluminescent imaging (BLI) to improve and refine traditional murine models of tumor growth and metastasis," Clinical and Experimental Metastasis, vol. 20, no. 8, pp. 733-744, 2003.

[31] F. Arguello, R. B. Baggs, and C. N. Frantz, "A murine model of experimental metastasis to bone and bone marrow," Cancer Research, vol. 48, no. 23, pp. 6876-6881, 1988.

[32] R. A. Barkhordar, E. D. Berston, and D. M. Ramos, "Cervical lymph node metastasis: model for study of Head/Neck melanoma," European Journal of Cancer Part B, vol. 31, no. 1, pp. 49-52, 1995.

[33] E. Frampas, C. Maurel, P. Thedrez, P. Remaud-Le Saëc, A. Faivre-Chauvet, and J. Barbet, "The intraportal injection model for liver metastasis: advantages of associated bioluminescence to assess tumor growth and influences on tumor uptake of radiolabeled anti-carcinoembryonic antigen antibody," Nuclear Medicine Communications, vol. 32, no. 2, pp. 147-154, 2011.

[34] H.-T. Song, E. K. Jordan, B. K. Lewis et al., "Rat model of metastatic breast cancer monitored by MRI at 3 tesla and bioluminescence imaging with histological correlation," Journal of Translational Medicine, vol. 7, article 88, 2009.

[35] M. Nogawa, T. Yuasa, S. Kimura et al., "Monitoring luciferaselabeled cancer cell growth and metastasis in different in vivo models," Cancer Letters, vol. 217, no. 2, pp. 243-253, 2005. 


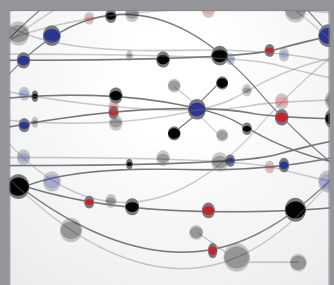

The Scientific World Journal
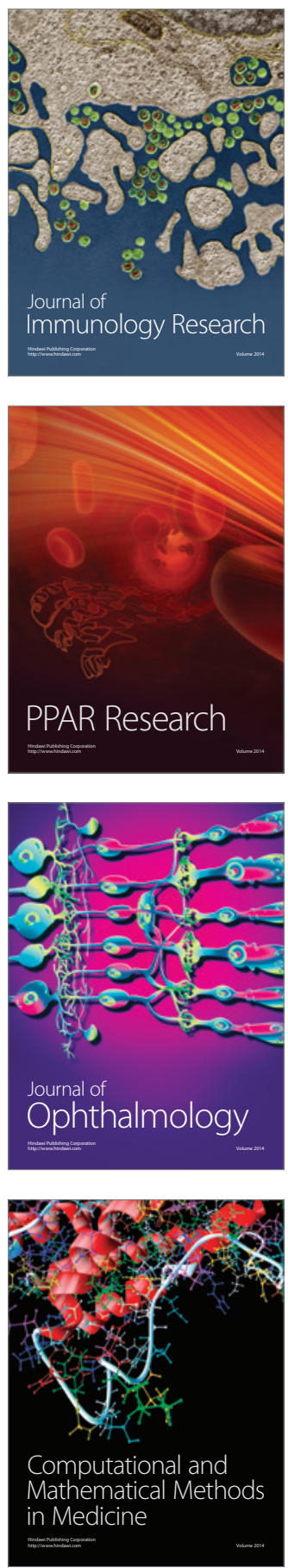

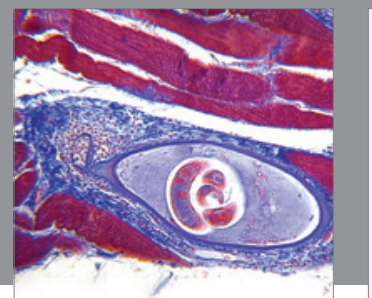

Gastroenterology

Research and Practice
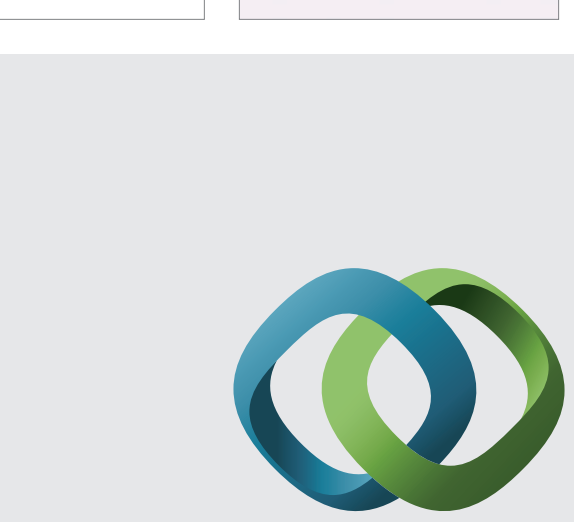

\section{Hindawi}

Submit your manuscripts at

http://www.hindawi.com
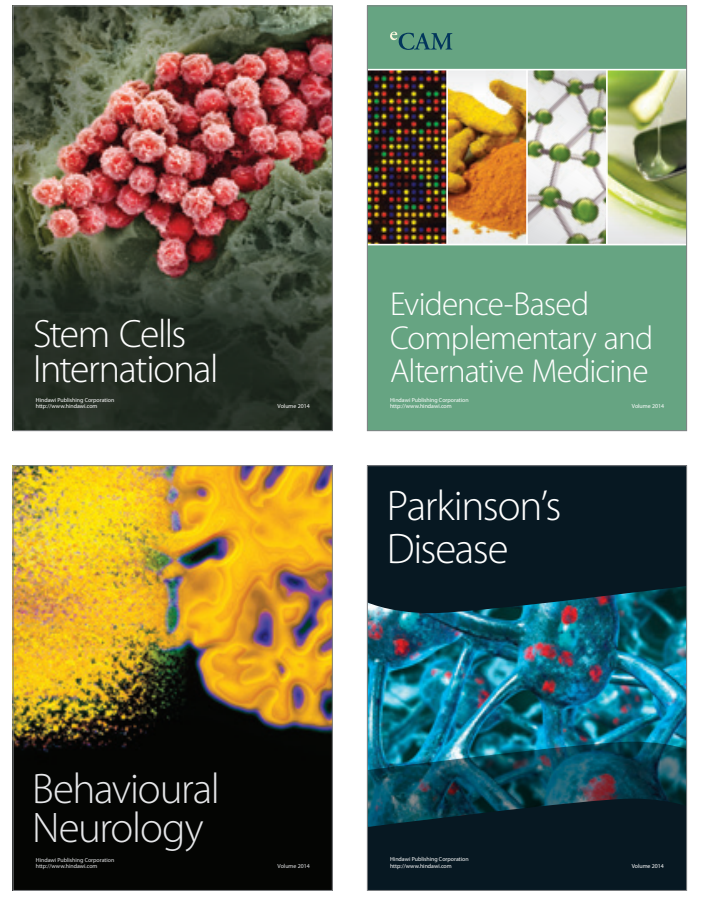
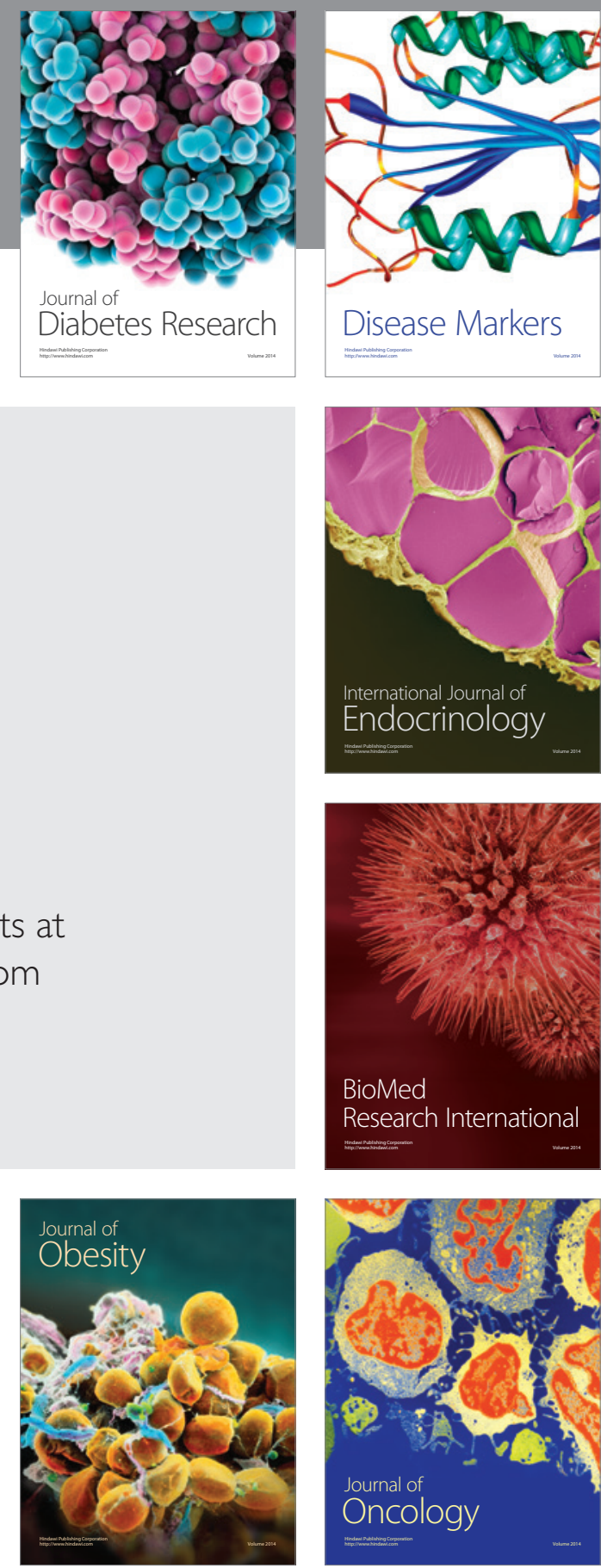

Disease Markers
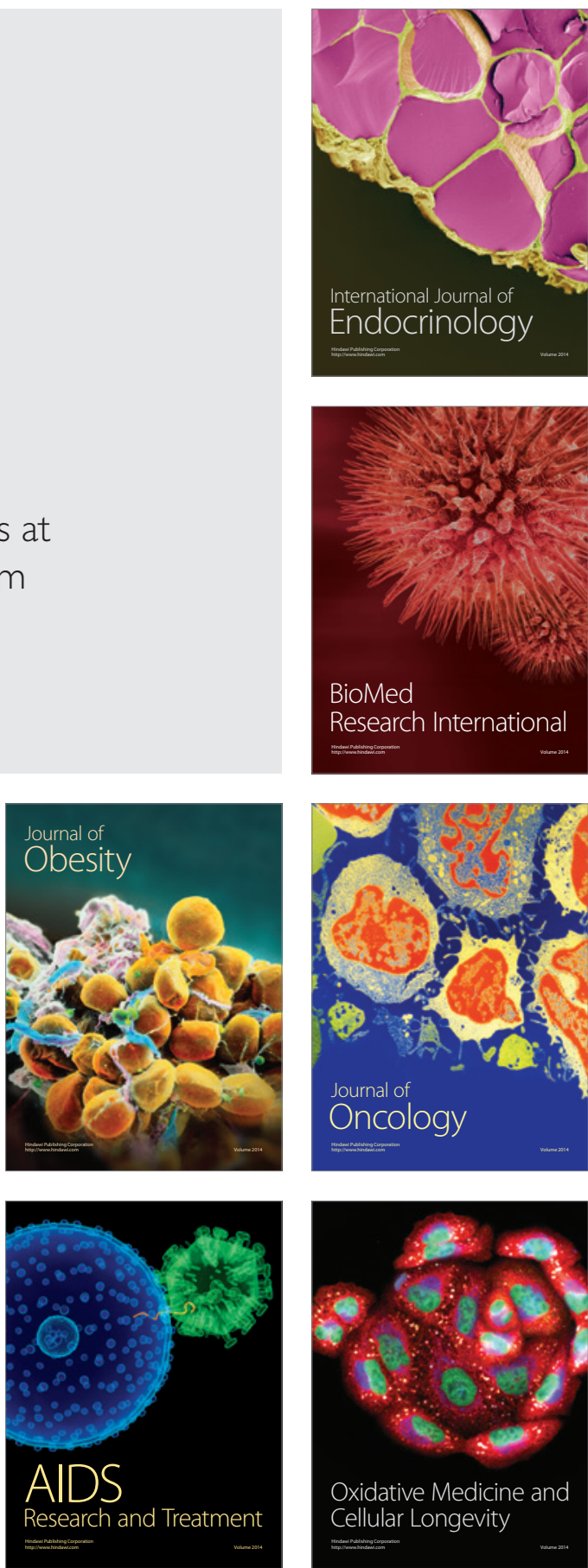